\section{DGI-021 COST-EFFICACY ANALYSIS OF ABIRATERONE FOR THE TREATMENT OF HORMONE-REFRACTORY METASTATIC PROSTATE CANCER PATIENTS}

doi:10.1136/ejhpharm-2013-000276.287

A Alcobia, A Soares. Hospital Garcia de Orta, Pharmacy, Almada, Portugal

Background In combination with prednisone or prednisolone, abiraterone is indicated for the treatment of patients with hormonerefractory metastatic prostate cancer (mHRPC) previously treated with a docetaxel-containing regimen. Abiraterone was evaluated in a phase 3, randomised, double-blind, placebo-controlled study.

Purpose To evaluate the cost-efficacy of abiraterone for the treatment of patients with mHRPC previously treated with a docetaxelcontaining regimen, using best supportive care as a comparator.

Materials and Methods Abiraterone efficacy and safety data were sourced directly from the above-mentioned phase 3 study. Two different efficacy parameters were considered: overall survival (OS) and progression free survival (PFS). The costs of the therapeutic options were calculated based on the direct cost of the drugs and the treatment duration described in the study. This study was conducted from an institutional perspective - the hospital perspective. Results In the phase III trial considered, the median OS was 14.8 months with abiraterone and 10.9 months with placebo. The median PFS was 10.2 months in the abiraterone group and 6.6 months in the placebo group. Median treatment duration was eight months for abiraterone and four months for placebo. The marginal efficacy for abiraterone is 3.9 months for OS and 3.6 months for PFS. Considering OS as efficacy parameter, the incremental costefficacy ratio (ICER) calculated for the two treatments is $€ 89.848$. When PFS is considered, the ICER calculated is $€ 97.336$.

Conclusions Based on this analysis, the ICERs calculated for abiraterone are too high for it to be considered a cost-effective option in the treatment of mHRPC when compared with mitoxantrone, in patients patients previously treated with a docetaxel-containing regimen.

No conflict of interest.

\section{DGI-022 COST-EFFICACY ANALYSIS OF CABAZITAXEL FOR THE TREATMENT OF HORMONE-REFRACTORY METASTATIC PROSTATE CANCER PATIENTS}

doi:10.1136/ejhpharm-2013-000276.288

A Alcobia, A Soares. Hospital Garcia de Orta, Pharmacy, Almada, Portugal

Background In combination with prednisone or prednisolone, cabazitaxel is indicated for the treatment of patients with hormonerefractory metastatic prostate cancer (mHRPC) previously treated with a docetaxel-containing regimen. Cabazitaxel was evaluated versus mitoxantrone in an open-label randomised phase III trial, the TROPIC study.

Purpose To evaluate the cost-efficacy of cabazitaxel for the treatment of patients with mHRPC previously treated with a docetaxelcontaining regimen, using mitoxantrone as a comparator.

Materials and Methods Cabazitaxel and mitoxantrone efficacy and safety data were sourced directly from the TROPIC trial. Two different efficacy parameters were considered: overall survival (OS) and progression free survival (PFS). The costs of the two therapeutic options were calculated based on the direct cost of the drugs, treatment duration and the probability of using granulocyte colonystimulating factors (filgrastim). This study was conducted from an institutional perspective - the hospital perspective.

Results In the TROPIC trial, the median OS was 15.1 months with cabazitaxel and 12.7 months with mitoxantrone, and median PFS was 2.8 months in the cabazitaxel group and 1.4 months in the mitoxantrone group. Median number of treatment cycles was six for cabazitaxel and four for mitoxantrone. The most frequent clinically significant grade $3 / 4$ adverse events were neutropenia (cabazitaxel $(82 \%)$ vs. mitoxantrone $(58 \%))$. The marginal efficacy of cabazitaxel vs. mitoxantrone is 2.4 months for OS and 1.4 months for PFS. Considering OS as efficacy parameter, the incremental costefficacy ratio (ICER) calculated for the two treatments is $€ 147.389$. When PFS is considered, the ICER calculated is $€ 248.871$.

Conclusions Based on this analysis, the ICERs calculated for cabazitaxel are too high for it to be considered a cost-effective option in the treatment of mHRPC, when compared with mitoxantrone, in patients previously treated with a docetaxel-containing regimen.

No conflict of interest.

\section{DGI-023 DESCRIPTION OF OMALIZUMAB USE FOR THE TREATMENT OF ASTHMA AFTER FOUR YEARS OF EXPERIENCE}

doi:10.1136/ejhpharm-2013-000276.289

L Serrano De Lucas, A Bustinza Txertudi, B Baza Martinez, S Sautua Larreategui, A De Basagoiti Gorordo, I Bilbao Meseguer, A Belaustegui Foronda, Z Baskaran Kaltzakorta, J Hernandez Goicoechea, E Rodriguez España. Hospital Universitario Cruces, Pharmacy, Barakaldo, Spain

Background Omalizumab's labelled indication is the treatment of IgE-mediated asthma. It has been used in our hospital since 2008. In 2011 it became necessary to develop a protocol that clarified patient selection and criteria for withholding treatment.

Purpose To describe the patients treated with omalizumab, focusing on whether they match our protocol's use criteria or not.

Materials and Methods All patients treated with omalizumab for asthma in our hospital were included. Data were obtained in October 2012 from electronic clinical records: treatment period, patient smoker or not, other medicines for asthma, basal IgE, adherence to treatment, omalizumab dosage and hospitalizations and emergency department visits before and after treatment.

Our omalizumab use protocol states these patient selection criteria: uncontrolled severe asthma with previous optimised therapy, basal IgE $>76 \mathrm{IU} / \mathrm{mL}$ and at least three emergency department visits or one hospitalisation in the previous year. Treatment withholding criteria are: evaluation after 16 weeks and stop if treatment shows no benefit.

Two different pharmacists examined each patient's information to establish if treatment was being effective and whether the hospital's protocol was being followed.

Results 31 patients were studied, 7 children and 24 adults. Treatment was stopped in 9 patients, due to lack of efficacy in 8 of them and to adverse effects in the other (diarrhoea, fever and skin reaction). Previous treatments included montelukast or theophylline in 19 patients (61\%). Basal IgE was below $76 \mathrm{IU} / \mathrm{mL}$ in one patient. Median duration of treatment was 637 days (72-1624). Regarding patients' adherence to treatment, $23 \%$ of patients missed a dose, $13 \%$ missed two and $6 \%$ missed three or more. 13 patients had had no pre-treatment hospitalizations or emergency department visits.

Treatment was evaluated as effective in 14 of the 22 patients who continued receiving omalizumab (64\%).

Conclusions Our patients still need to be selected better. Protocol compliance is lower than desirable.

No conflict of interest.

DGI-024 DEVELOPMENT OF A GUIDE FOR ADMINISTERING ANTIVIRAL DRUGS BY GASTROSTOMY OR NASOGASTRIC TUBE

doi:10.1136/ejhpharm-2013-000276.290

J Arenas Villafranca, M Nieto Guindo, C Lopez Martin, R Romero Dominguez, ME Blanco Rivas, M Garrido Siles. 'Empresa Pública Hospital Costa del Sol, Farmacia Y Nutrición, Marbella, Spain 